FERMILAB-Pub-92/320-E

$\mathbf{E 6 8 7}$

\title{
A Wide Band Photon Beam at the Fermilab Tevatron to Study Heavy Flavors
}

\author{
P.L. Frabetti et al \\ The E687 Collaboration \\ Fermi National Accelerator Laboratory \\ P.O. Box 500, Batavia, Illinois 60510
}

November 1992

Approved for publication in Nuclear Instruments and Methods in Physics Research - Section A 


\section{Disclaimer}

This report was prepared as an account of work sponsored by an agency of the United States Government. Neither the United States Government nor any agency thereof, nor any of their employees, makes any warranty, express or implied, or assumes any legal liability or responsibility for the accuracy, completeness, or usefulness of any information, apparatus, product, or process disclosed, or represents that its use would not infringe privately owned rights. Reference herein to any specific commercial product, process, or service by trade name, trademark, manufacturer, or otherwise, does not necessarily constitute or imply its endorsement, recommendation, or favoring by the United States Government or any agency thereof. The views and opinions of authors expressed herein do not necessarily state or reflect those of the United States Government or any agency thereof. 


\title{
A wide band photon beam at the Fermilab Tevatron to study heavy flavors
}

\author{
P.L. Frabetti \\ Dip. di Fisica dell'Universit and INFN - Bologna, I-40126 Bologna, Italy \\ C.W. Bogart ${ }^{1}$ J. Cumalat, S. Culy, H. Cheung, W. Johns \\ University of Colorado, Boulder, CO 80308, USA
}

J.N. Butler, S. Cihangir, R. Currier, F. Davenport ${ }^{2}$, I. Gaines, P.H. Garbincius, S. Gourlay, D.J. Harding, R. Justice, P. Kasper, A. Kreymer, P. Lebrun, S. Shukla Fermilab, Bateria, IL 60510, USA

S. Bianco, F. L. Fabbri, A. Spallone, A. Zallo Laboratori Nasionali di Frascati, I-00044 Frascati, Italy

G. Jaross ${ }^{3}$, K. Lingel ${ }^{4}$, J. Wiss

Uniretsity of Illinois at Urbana-Champaign, Urbana,IL 61801

G. Alimonti, G. Bellini, B. Caccianiga, W.R. Cavaletti, L. Cinquini ${ }^{5}$, P. D'Angelo, M. Di Corato, M. Giammarchi, P. Inzani, F. Leveraro,

S. Malvezzi, P.F. Manfredi ${ }^{\circ}$, D. Menasce, E. Meroni, L. Moroni, D. Pedrini,

L. Perasso, F. Ragusa, A. Sala, S. Sala, D. Torretta ${ }^{5}$, M. Vit tone ${ }^{5}$

Dip. di Fisice dell'Unirerait and INFN - Milano, I-20133 Milan, Italy

D. Buchholz, C. Castoldi ${ }^{7}$, D. Claes ${ }^{8}$, B. Gobbi, S. Park ${ }^{5}$, R. Yoshida ${ }^{8}$ Northwestern University, Evanston, IL 60208 , USA

J.M. Bishop, J.K. Busenitz ${ }^{10}$, N.M. Cason, J.D. Cunningham ${ }^{11}$, R.W. Gardner ${ }^{12}$, C.J. Kennedy, E.J. Mannel ${ }^{13}$, R.J. Mountain, D.L. Puseljic,

R.C. Ruchti, W.D. Shephard, Z.Y. Wu, M.E. Zanabria University of Notre Dame, Notre Dame, IN 46556, USA

V. Arena, G. Boca, R. Diaferia, S.P. Ratti, C. Riccardi, P. Vitulo Dip. di Fisica Nucleare e Teorica dell'Unirersite and INFN - Pavia, I-27100 Povia, Itaiy

A. Lopez, University of Puerto Rico at Mayaguez, Puerto Rico

H. Mendez ${ }^{14}$, Cinvestar-IPN, A.P. 14-740,07000 Mexico DF, Mexico and Fermilab

J. R. Wilson, Unirersity of South Carolina

November 5, 1992 

${ }^{1}$ Present address: Vector Research Company, 6903 Rockledge Drive, Bethesda, MD 20817, USA.

${ }^{2}$ Present address: University of North Carolina-Asheville, Asheville, NC 28804, USA.

${ }^{3}$ Present address: STX Inc., 4400 Forbes Blvd., Lanham, MD 20706, USA.

${ }^{4}$ Present address: University of Colorado, Boulder, CO 80309, USA.

5 Present address: Fermilab, Batavia, IL 60510, USA.

B Present address: Dipartimento di Elettronica, Università di Pavia, Pavia, Italy.

7 Present address: Dip. di Fisica Nucleare e Teorica dell'Università and INFN - Pavia, I-27100 Pavia, Italy.

8 Present address: State University of New York, Stony Brook, NY 11794, USA.

- Present address: NIKHEF-H, 1009 DB, Amsterdam, The Netherlands.

${ }^{10}$ Present address: University of Alabama, Tuscaloosa, AL 35487, USA.

11 Present address: Brandeis University, Waltham, MA 02254, USA.

12 Present address: University of Illinois at Urbana-Champaign, Urbana, IL 61801, USA.

${ }^{13}$ Present address: Nevis Labs, Columbia University, Irvington, NY 10533, USA.

${ }^{14}$ Present address: University of Illinois at Chicago, Chicago, IL 60680, USA. 
For many years, the use of high energy photon beams has been recognized as a good technique for the investigation of charmed particles[1]. The cross section is a relatively large fraction (approximately $1 \%$ ) of the total hadronic cross section at high energies. The challenge was to develop a high energy and high intensity beam that produces enough charmed particles to obtain very high statistics so that one can answer detailed questions about charm production and decays. The Fermilab Wideband Photon Beam is an attempt to meet that challenge.

The beamline configuration and capabilities have evolved since the test run in 1985 and the first physics run in 1987-88. For the 1990-91 data run, improvements were made to increase the photon yield and to improve the photon energy tagging resolution. Althought the main emphasis of this report is on the original 1985-88 beam configuration, two fully operational upgrades for the 1990-91 run, namely the incident electron beam momentum tagging spectrometer and the $L D_{2}$ primary beam target, are described. The beam was also given the capability of transporting simultaneously $e^{+}$and $e^{-}$beams. The performance of this 'double-band' configuration was studied briefly duing the 1990-91 running period and will be described in a future publication. A description of the Fermilab E687 Spectrometer utilizing this photon beam facility has recently been published[2].

\section{Description, Yields, and Backgrounds}

At a proton accelerator, photons are derived indirectly from the strong interactions. One must solve two problems in order to make a clean photon beam: first, one must collect particles produced over a wide range of momenta and angles to form the beam; second, one must remove all the unwanted particles which are produced together with the photons. The unwanted particles include charged hadrons, $\pi^{+}, \pi^{-}, K^{+}, K^{-}, \mathrm{p}$, and $\bar{p}_{1}$ as well as neutral particles such as neutrons and $K_{L}^{0}$ 's.

The photon beam is produced by the conventional "bremsstrahlung method" $[3,4]$ as follows:

1. $800 \mathrm{GeV} / \mathrm{c}$ protons interact in a target, the "primary production target". Neutral pions are created and decay into two photons. Immediately downstream of the target, sweeping magnets remove all the charged particles. The photons emerge from the target box at zero degrees. 
2. The photons then interact with a lead foil, the "converter", where they produce electron-positron pairs.

3. The electrons are then captured by a conventional beam transport consisting of dipole magnets and quadrupoles and are bent away from zero degrees. Neutral hadrons, neutrons and $K_{L}^{0}$ 's, along with unconverted photons, travel forward at zero angle and are absorbed in a "neutral dump".

4. The electron beam is transported around the dump, refocussed, and passed through a thin lead foil, the "radiator", to produce photons by bremsstrahlung. The electrons are then deflected off to the side by dipole magnets, the "electron sweepers", and into a dump, the "electron dump", while the photons pass straight ahead to the experimental target.

Figure 1 shows schematically the various steps required to produce a photon beam by this method. Figure 2 shows a schematic of the charged particle transport employed in the Wideband Photon Beam.

The photons produced by this method emerge from a chain of three interactions and one decay process: the primary interaction produces a $\pi^{0}$ which then decays into two photons; a conversion of one of the photons to an electronpositron pair; and the bremsstrahlung of the electron to a photon and an electron. The $\pi^{0}$ decay, the pair conversion and the bremsstrahlung interaction, all degrade the final energy of the photon which emerges from the end of the chain. The photon beam is thus a tertiary beam and it is difficult, using this method, to achieve high intensity, especially at high photon energies. The advantage of this technique, however, is that it is relatively free from hadronic background compared to other methods. This is discussed in more detail below.

In order to achieve intense beams of high energy photons, it is crucial to collect electrons emerging from the converter over a very large range of angles and momenta. Since the electrons are charged, they may be collected by quadrupoles. It is possible to achieve large angular acceptance in this kind of beam while achieving a reasonably small beam spot on the experimental target. The optical beam transport system of the Wideband Beam is arranged to collect electrons with a range of $\pm 15 \%$ around a 'central' momentum setting.

The dipole arrangement in the secondary beam is a 'double dogleg'. It has two important features: first, there are no dipoles before the first set of quadrupoles and the bend angles in the dipole string in the center of the beam cancel each other so that there is no net first order momentum dispersion in 


\section{Bremsstrahlung Photon Beam}

Step 1: Produce a Neutral Beam

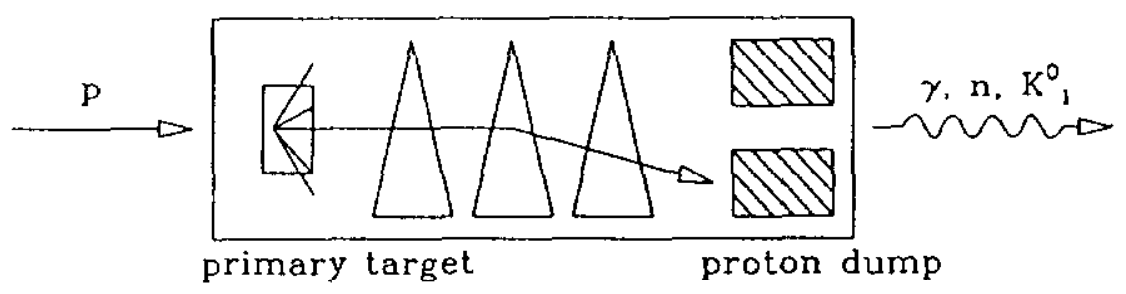

Step 2: Convert Photons

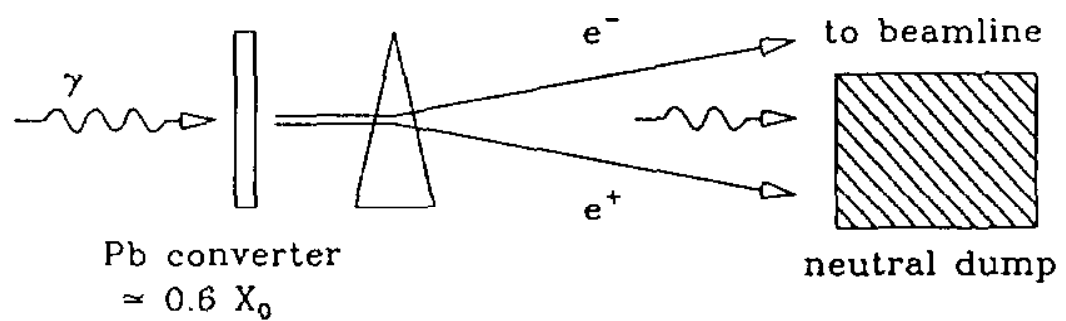

Step 3: Capture and Transport Electrons

Step 4: Radiate Photons

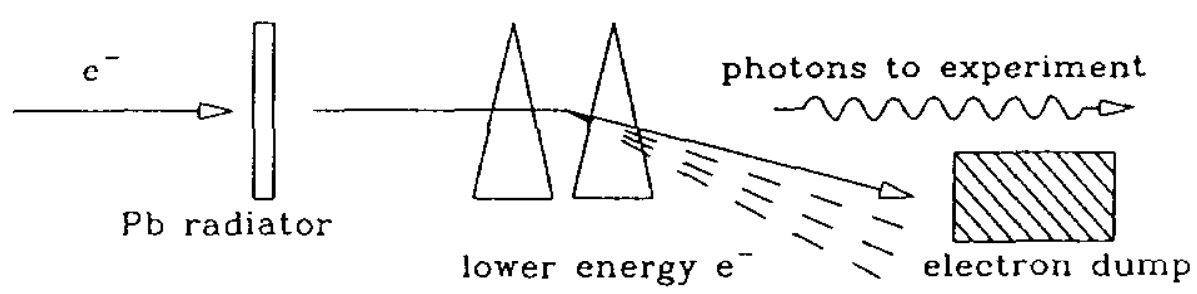

Figure 1: Steps required to produce a bremsstrahlung photon beam. 

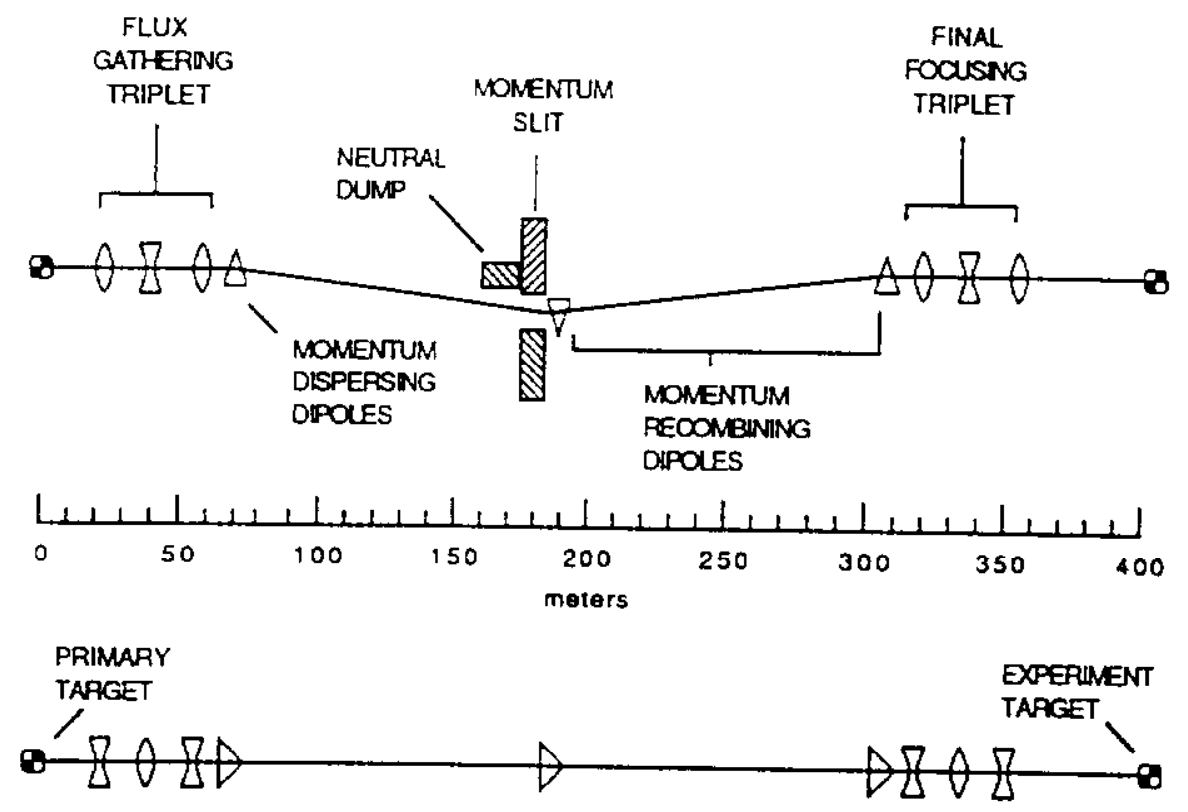

Figure 2: Charged particle transport for the Fermilab Wideband Photon Beam. Top is plan view. Bottom is elevation view.

the downstream quadrupole section of the beam; second, all the bends are very small. The first feature keeps the beam relatively small as it passes through the quadrupoles. The second preserves the momentum acceptance in the double dogleg itself. The dipoles are all of the Fermilab '6-3-120' [5] type and have apertures which are 6 inches $\times 3$ inches.

In the upstream part of the beam, there is a flux-gathering symmetric quadrupole triplet, consisting of standard Fermilab '4Q120' magnets[6], which have 4 inch apertures. The field gradients are chosen to focus the low momentum components at the momentum slit in the middle of the double dogleg both vertically and horizontally. The high momentum components are not well focussed and are essentially parallel near the center of the dogleg. At the downstream end of the beam, another symmetric quadrupole triplet of 4Q120 magnets images both the highest and lowest momentum components of the beam onto the experimental target. Thus, in conventional terms, the beam is a 'two-stage' beam or 'point-to-point-to-point' for the low momentum components but is a 'one stage beam' with 'point-to-parallel-to-point' optics for the higher momentum components. The intermediate momentum components are not completely refocussed at the target of the experiment. It is obvious that, while this arrange- 
Table 1: Wide Band Beam Properties.

\begin{tabular}{|l|l|}
\hline Horizontal spot size at production target & $\delta x= \pm 1 \mathrm{~mm}$ \\
Vertical spot size at production target & $\delta y= \pm 1 \mathrm{~mm}$ \\
Geometric horizontal angle accepted & $\delta \theta_{x}= \pm 1.0 \mathrm{mrad}$ \\
Geometric vertical angle accepted & $\delta \theta_{y}= \pm 0.75 \mathrm{mrad}$ \\
Geometric solid angle accepted & $\Delta \Omega=6.0 \mu s t e r a d$ \\
Maximum momentum bite & $\frac{\Delta p}{p} \approx \pm 15 \%$ \\
Effective acceptance & $\Delta \Omega \times \frac{\Delta p}{p} \approx 96 \mu \mathrm{ster} \%$ \\
Horizontal spot at experimental target & $\delta x= \pm 1.25 \mathrm{~cm}$ \\
Vertical spot at experimental target & $\delta y= \pm 0.75 \mathrm{~cm}$ \\
Horizontal divergence at experimental target & $\delta \theta_{x}= \pm 0.6 \mathrm{mrad}$ \\
Vertical divergence at experimental target & $\delta \theta_{y}= \pm 0.5 \mathrm{mrad}$ \\
\hline
\end{tabular}

ment produces high acceptance, it will not produce a small beam spot at the experimental target because most of the momentum components of the beam are not truly imaged at the target.

The parameters of the Wide Band Beam are shown in Table 1. The photon beam spot has a half-width of greater than a centimeter at the base. This is a disadvantage for a neutral beam in that event vertex reconstruction must rely soley on the experimental spectrometer. However, radiation damage, singles rates, and coincident backgrounds are spread over a wider area of the vertex silicon strip detectors.

The method by which the optics is arranged to achieve both large momentum and angle acceptance has been explained. Additional parameters, such as the material and thickness of the production target, the converter, and the radiator, influence the final photon flux that can be achieved. Each has an 'optimum' value. One cannot make the production target too long because photons can convert as they travel from the production point to the end of the target. The converter cannot be made too thick because the electrons radiate energy via bremsstrahlung as they travel through the radiator from the production point. Increasing the converter beyond a certain thickness will produce more electrons but will soften the energy spectrum. The result is a reduction in the number of the relatively higher energy range of the photon spectrum. Finally, if the radiator is made too thick, many of the electrons give rise to more than one photon. This can make the interpretation of the events more difficult and can create rate problems in the detectors of the experiment.

A full calculation of the flux must include all these effects and is carried out by Monte Carlo techniques. Based on the Monte Carlo calculation, the 
production target is chosen to be $18 \mathrm{in}$. of beryllium and the converter is chosen to be $50 \%$ of a radiation length of lead. The radiator is chosen to be $20 \%$ of a radiation length of lead, based on studies of the effect of multiple bremsstrahlung on the performance of the experimental apparatus, especially of the microstrip detector.

Figure 3 shows the measured electron yields per incident $800 \mathrm{GeV} / \mathrm{c}$ proton and compares them to the Monte Carlo calculation. For energies above 250 $\mathrm{GeV}$, measured values are within $15 \%$ of the calculated values, consistent with the systematic uncertainties of the measurements and extrapolations used in the calculation. The photon spectrum obtained with a $350 \mathrm{GeV} / \mathrm{c}$ momentum $e^{-}$beam is shown in the next section.

One major reason for using a bremsstrahlung beam is to reduce the hadronic background in the beam. The sources of this background are neutrons and $K_{L}^{0}$ 's in the beam. These neutral hadrons escape the target box along with the photons. When they strike the converter, a small fraction interacts and may produce high energy charged hadrons which are captured by the secondary (electron) beam transport. If the secondary beam is set up to transport negatively charged particles, the dominant background is $\pi^{-}$. If the secondary beam is set to transport positive particles, then the background is a mixture of $\pi^{+}$and protons and is typically higher than for the negative setting. The relative number of protons also rises as the secondary beam energy approaches the primary beam energy. This increasing proton background was determined to be from the decay of leading $\Lambda^{0}$ 's which decay downstream of the target box sweeping magnets. For these reasons, one always chooses to operate the secondary beam so as to capture and transport negative particles. The charged hadrons eventually arrive at the 'radiator' where they may undergo hadronic interactions. These hadronic interactions are only a problem if they produce neutral particles at such small angles that they eventually strike the experimental target. This can occur if the are produced within milliradian of the incident charged hadron. Any charged particles produced in these collisions are removed by the 'electron sweepers' just downstream of the radiator.

Hadronic background comes from a sequence of events each of which has relatively low probability. The resultant background of neutral hadrons at the experimental target is, both from Monte Carlo calculation and from measurement, $10^{-5}$ neutrons per photon. Less than $1 \%$ of the hadronic final state events written to tape are produced by this neutral hadron beam background.

The beam can be operated in a variety of modes to help calibrate experimen- 


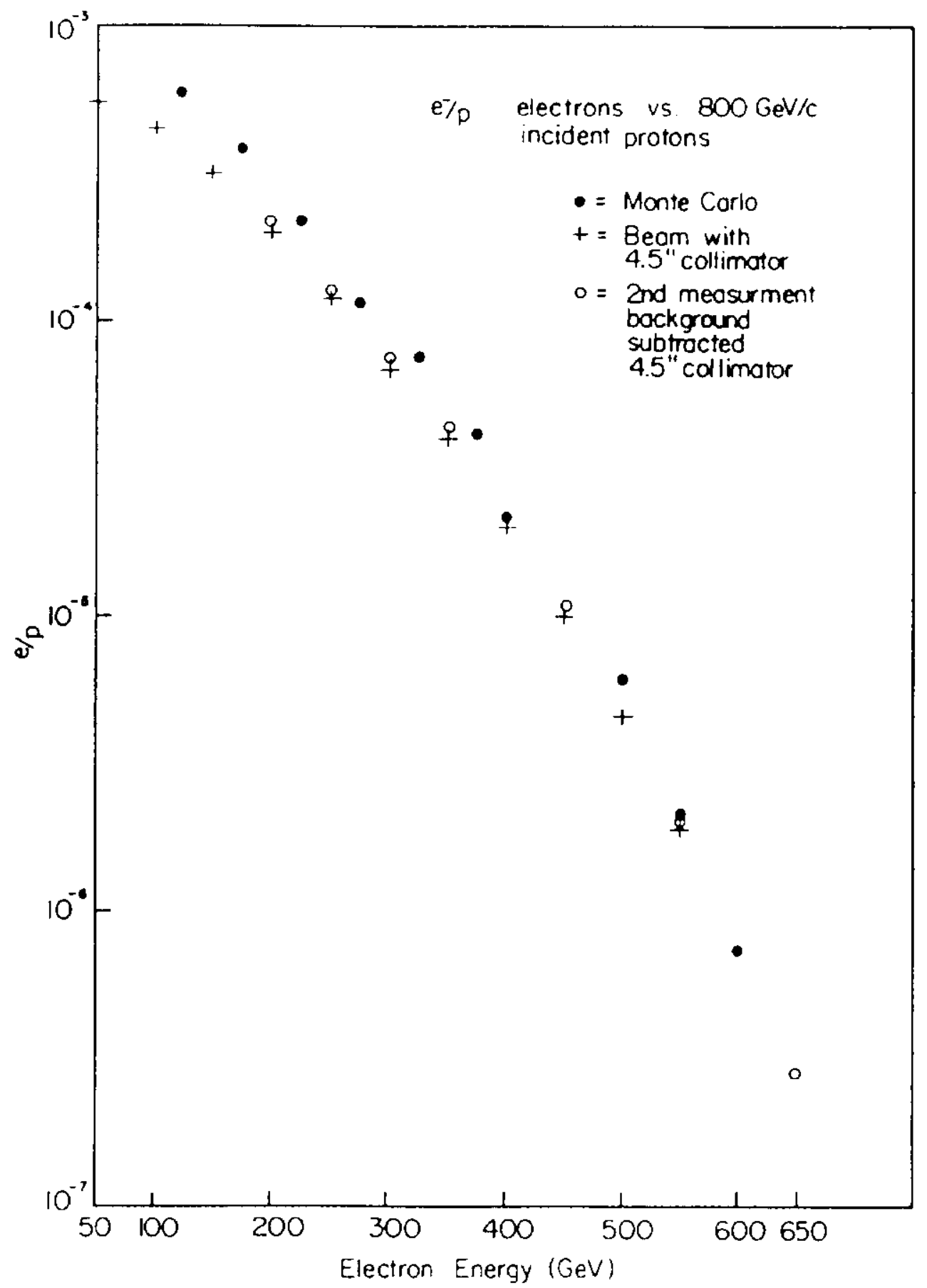

Figure 3: Electron yield per incident $800 \mathrm{GeV}$ proton as a function of electron energy. 


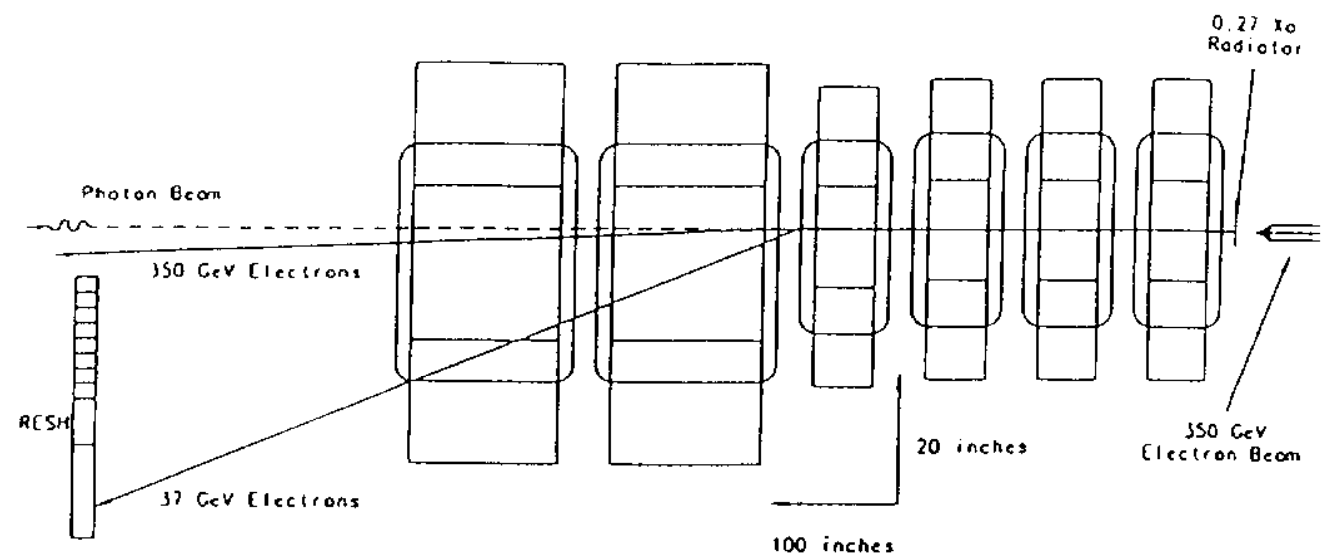

Figure 4: Schematic of Recoil Electron Tagging System.

tal equipment. By turning the electron sweepers off and removing the radiator, one can bring the electron bean directly into the experiment and use it to calibrate the electromagnetic calorimeters. By inserting a lead absorber into this 'electron beam', one can remove the electrons and obtain a $\pi^{-}$beam which can be used to calibrate the calorimeters. If a 10 foot long steel absorber is moved into the beam near the upstream end, all the electrons and pions are absorbed. In this case, the dominant flux through the detector consists of muons which escape from the primary target box. This so-called 'muon beam' is composed of high energy muons travelling at small angles to the central axis of the beam and spread out over the whole detector. It can be used for detector alignment, chamber efficiency studies, muon detector calibration, and various other purposes.

\section{Beam Tagging and Beam Gamma Monitor}

The Wideband Beam has a simple photon energy tagging system. It is shown schematically in Fig. 4. Its purpose is to determine the energy of the interacting photon on an event-by-event basis. It also serves the function of requiring a selectable minimum electron energy loss in the radiator in the Level II trigger to effectively "harden" the bremsstrahlung spectrum.

The incident electron, momentum selected by the Wideband Beam, strikes a lead radiator and produces photons by bremsstrahlung. After this energy loss, the electron is momentum analyzed in a magnetic spectrometer with the Radiated Electron Shower Hodoscope (RESH) as detector. Electrons are iden- 


\begin{tabular}{|c|c|c|c|c|}
\hline $\begin{array}{l}\text { Counter } \\
\text { Name }\end{array}$ & $\begin{array}{l}\text { Nominal } \\
\text { Photon } \\
\text { Tag } \\
(\mathrm{GeV})\end{array}$ & $\begin{array}{l}\text { Centerline } \\
\text { Magnetic } \\
\text { Deflection } \\
\text { (in.) }\end{array}$ & $\begin{array}{l}\text { Nominal } \\
\text { Width } \\
\text { (in.) }\end{array}$ & $\begin{array}{l}\text { \# Layers, Material, } \\
\text { and Thickness }\end{array}$ \\
\hline $\begin{array}{l}\text { non- } \\
\text { Radiating } \\
\text { Electrons }\end{array}$ & 0 & 4.03 & & \\
\hline RESH 1 & 138 & 7.37 & 2.5 & $23 \mathrm{~Pb}$, Lucite, $1 / 4$ in. each \\
\hline RESH 2 & 205 & 9.98 & 2.5 & $n$ \\
\hline RESH 3 & 235 & 12.56 & 2.5 & $"$ \\
\hline RESH 4 & 254 & 15.14 & 2.5 & $n$ \\
\hline RESH 5 & 268 & 17.72 & 2.5 & $"$ \\
\hline RESH 6 & 278 & 20.30 & 2.5 & $"$ \\
\hline RESH 7 & 286 & 22.88 & 2.5 & $"$ \\
\hline RESH 8 & 293 & 25.46 & 2.5 & $n$ \\
\hline RESH 9 & 302 & 29.79 & 6.0 & $20 \mathrm{~Pb}$, Acrylic Scint, $1 / 4$ in. \\
\hline RESH 10 & 310 & 37.54 & 11.5 & $20 \mathrm{~Pb}$,Acrylic Scint, $1 / 4$ in. \\
\hline BGM & 0 & & $9 \times 9$ & $45, \mathrm{~Pb}$, Lucite, $1 / 8$ in. each \\
\hline
\end{tabular}

Table 2: Tagging Counter Specifications. The lead is stiffened with $6 \% \mathrm{Sb}$ by weight. The vertical (non-bend) size of the RESH counters is 6 in. The RESH 10 aperture is limited by the tagging magnet vacuum chamber wall.

tified by a large ratio of energy deposition to momentum (E/p) in this RESH hodoscope. Multiple bremsstrahlung may occur in the radiator. Typically, only one photon interacts in the experimental target. The sum of the energies of any non-interacting photons produced by that given electron is measured in a zerodegree shower counter, the Beam Gamma Monitor (BGM), located towards the end of the spectrometer. The physical properties of these detectors are given in Table 2.

The photon energy is calculated, on an event-by-event basis, from the following formula:

$$
E_{0}=E^{\prime}+k_{\text {interacting }}+\sum k_{\text {additional }}
$$

where: 


\begin{abstract}
$E_{0} \quad$ is the incident electron energy, $350 \mathrm{GeV} \pm 50 \mathrm{GeV}(\sigma)$. In the absence of measurement of the beam energy on an event-by-event basis, the incident electron is defined to have the nominal $350 \mathrm{GeV}$ beam energy.

$E^{\prime} \quad$ is the energy of the electron after radiation loss as measured in the magnetic spectrometer and RESH hodoscope.

$k_{\text {interacting }}$ is the energy of the photon interacting in the experimental target producing the hadronic event detected in the E-687 spectrometer.

$\sum k_{\text {additional }}$ is the sum of the energies of any additional multiple bremsstrahlung photons produced by the electron in the radiator (and either not interecting in experimental target or pair producing with the $e^{+} e^{-}$ pairs refocused into the BGM counter).
\end{abstract}

Then, $k_{\text {interacting }}=E_{0}-E^{\prime}-\sum k_{\text {additional }}$.

The uncertainty in each of these terms limits the resolution in the estimate of the energy of the interacting photon. The major limitation is due to the $\pm 50 \mathrm{GeV}$ energy spread of the incident electron beam. Beginning with the 1990 run, an incident beam magnetic spectrometer[7] measures the incident electron momentum to $\pm 2 \%$. This system is described in the next section.

A second major concern is the multiple bremsstrahlung in the thick radiator. Under most running conditions, a $0.20 X_{0} \mathrm{~Pb}$ radiator is used. There is an additional $0.07 X_{0}$ effective radiator due to nearby beam monitoring scintillation counters and titanium vacuum windows. Therefore, the total effective radiator is $0.27 X_{0}$.

\title{
2.1 Hardware
}

The magnetic field which bends the radiated electron is generated by six dipole magnets which were prototypes for the Fermilab Anti-proton Accumulator Ring[8]. They produce a $\int B d l$ of 89.5 kilogauss-meters at an operating current of 1190 amperes. The distance from the effective bend point to the RESH plane (shower maximum) is 13.34 meters. The non-radiating nominal $350 \mathrm{GeV}$ electrons are deflected 10.2 centimeters from the photon beamline and strike a lead and steel dump. The dipole sweeping magnets are offset to the west to increase the horizontal aperture and good field region for the radiated 
electrons.

The RESH hodoscope counters are either lead-lucite or lead-acrylic scintillator shower counters of about $24 X_{0}$ depth. The RESH counters are outfitted with RCA 8575 photomultiplier tubes and transistorized bases [9].

The $\mathrm{BGM}$ is a lead-lucite shower counter [45 layers of $1 / 8$ inch $\mathrm{Pb}(+6 \%$ $\mathrm{Sb}$ ) and $1 / 8$ inch lucite] of $24 X_{0}$ depth with bent lucite light pipes leading to a Amperex 58AVP photomultiplier tube with a 9 stage resistor base [10]. Slight variations of the $B G M$ counter are used in later running periods to perform the same function.

The RESH counters and BGM have CAMAC-controlled pulsed LED's for performance and gain monitoring.

Since this tagging system is intended to operate at high rates, it is important to minimize the time resolution, and to have pulse pair resolutions of one RF bucket ( $18.8 \mathrm{nsec}$ ) if possible. The coincidence resolving time for the coincidence registers is approximately 4 nsec. Due to problems with the ECL gate driving circuit feeding the LRS 1885 ADC's, the minimum gate widths for RESH and BGM is $38 \mathrm{nsec}$, corresponding to two RF buckets.

\subsection{Use of RESH in Triggering}

The typical energy deposition in an individual RESH(i) counter, for events with the corresponding discriminator and coincidence register set, is shown in Fig. 5. There is a prominent peak due to the momentum analyzed radiated electron striking near the center of the individual RESH(i). There is a tail on the low energy deposition side due to transverse shower leakage for electrons striking that RESH near either of its side edges. There is also a tail extending upward from pedestal (not fully displayed since it is mostly below discriminator threshold) due to radiated electrons striking an adjacent RESH(i-1) or RESH( $(i+1)$ hodoscope cell with some energy deposited in RESH(i) due to transverse shower spread. Such shower sharing between adjacent RESH counters can be used to localize the electron impact point to considerably better resolution than the RESH counter width. Due to the resolution limitations inherent in the incident electron beam with such a large, untagged momemtum bite, a simpler tagging algorithm is used.

A discriminator threshold is set at approximately $20 \%$ of the nominal radiated electron peak for each RESH counter. This logic signal is latched in a coincidence register gated by the Level I trigger. The output of this latch is then sent to a LeCroy LRS 4516 Programmable Logic Unit (PLU). This PLU 


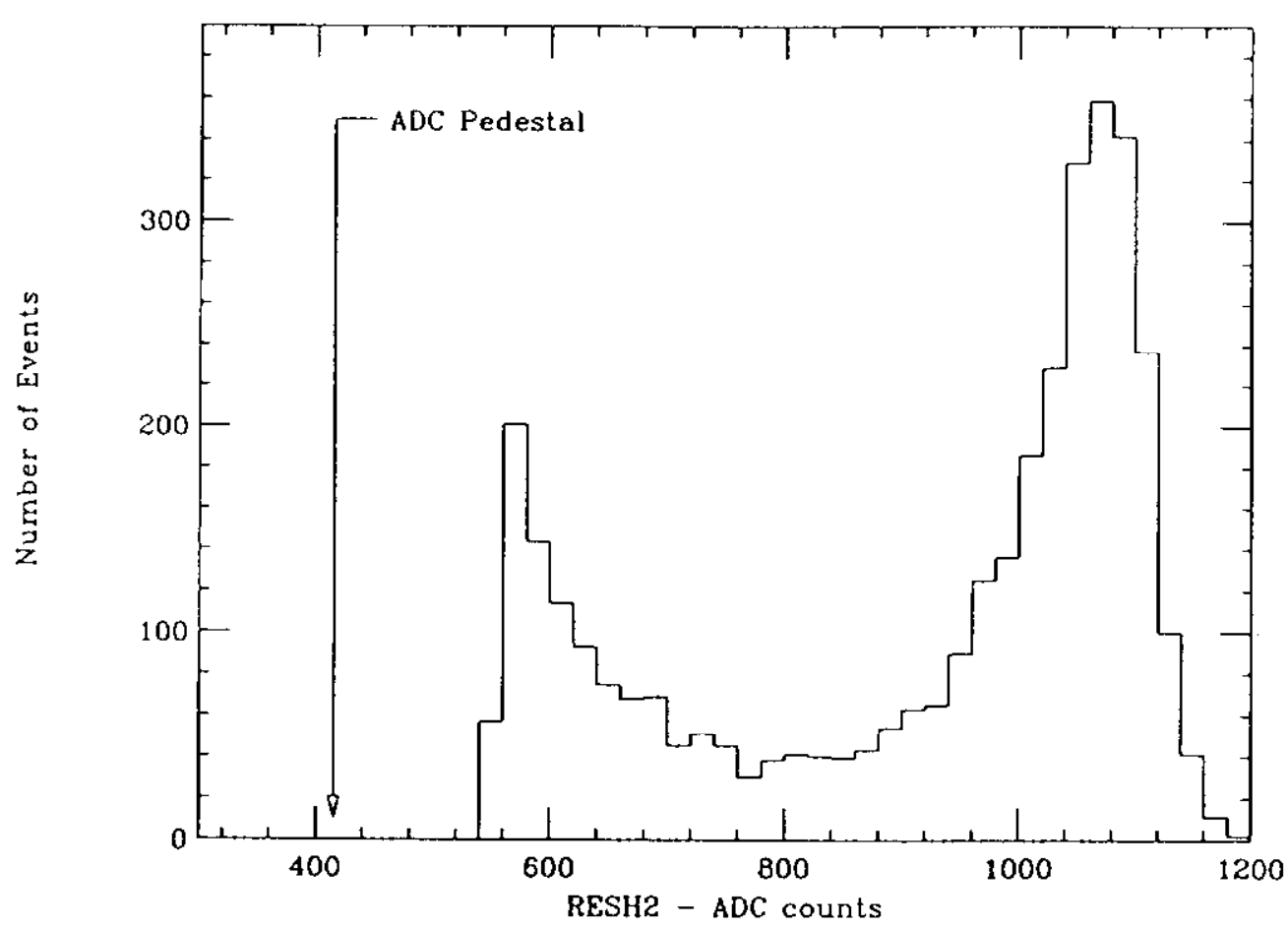

Figure 5: Typical RESH counter pulse height spectrum with discriminator and coincidence register set.

Table 3: RESH patterns considered valid by trigger logic.

\begin{tabular}{|c|c|c|}
\hline $\begin{array}{l}\text { or } \\
\text { or }\end{array}$ & $\begin{array}{l}\operatorname{RESH}(i) \\
\operatorname{RESH}(i) \times \operatorname{RESH}(i+1) \\
\operatorname{RESH}(i-1) \times \operatorname{RESH}(i) \times \operatorname{RESH}(i+1)\end{array}$ & $\begin{array}{l}\text { a single hit } \\
\text { two adjacent hits } \\
\text { three adjacent hits }\end{array}$ \\
\hline
\end{tabular}

then passes the RESH hit patterns to the Level II trigger logic, consistent with a single electron striking RESH. The valid patterns are shown in Table 3 . Since the triple adjacent hit pattern occurs less than $1 \%$ of the time, this triple pattern is cut in the offline analysis.

There are three levels of RESH signals passed from the RESH PLU to the Level II trigger corresponding to:

1- RESH-LOW nominal electron energy loss greater than $116 \mathrm{GeV}$

2- RESH-MED nominal electron energy loss greater than $193 \mathrm{GeV}$

3- RESH-HIGH nominal electron energy loss greater than $212 \mathrm{GeV}$

It is again noted that the electron energy loss represents the sum of the energies of the radiated photons. Most of the data is taken using the RESHLOW threshold.

The use of the patterns for the 10 single RESH hits and the 9 adjacent RESH hits provides 19 photon energy tagging bins. This is more than adequate since the difference between adjacent bins is typically smaller than the incident electron beam momentum uncertainty. 


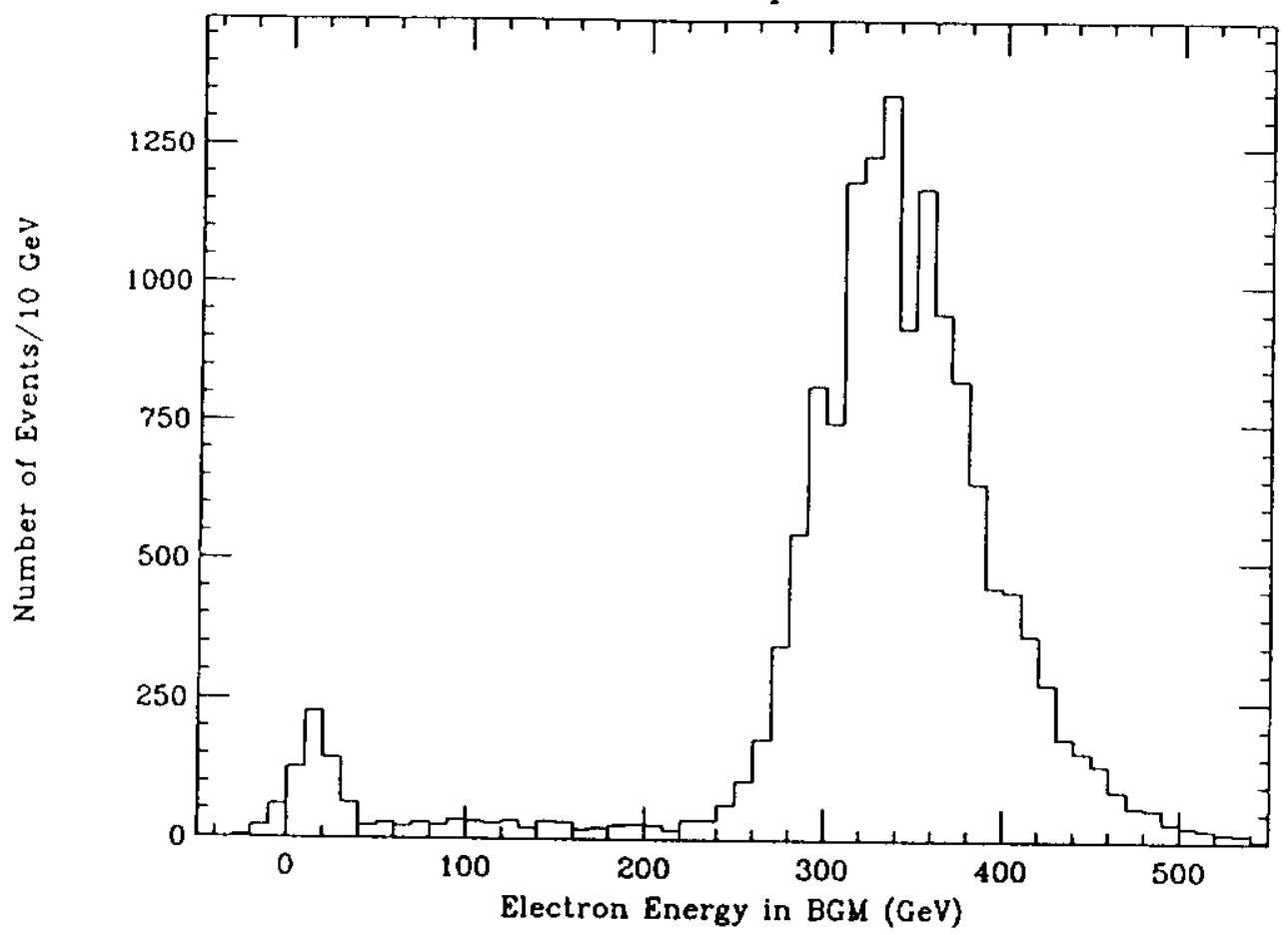

Figure 6: Electron beam energy distribution for $350 \mathrm{GeV}$ central beam setting as measured calorimetrically in the BGM counter.

\subsection{Performance}

Photon tagging system performance data are taken during dedicated runs where either an electron beam is taken directly to the BGM counter or a photon beam is used with a simple charged particle trigger with the spectrometer analyzing magnets OFF to allow converted pairs to strike the BGM. During these photon calibration runs, the energy deposited in BGM is technically the energy loss of the electron, $\Delta E=E_{0}-E^{\prime}$, which could be comprised of multiple photons. This allowes cross calibration and monitoring of the RESH and BGM systems. Figure 6 shows the electron beam energy distribution measured calorimetrically in the BGM shower counter. The photon beam energy distribution is shown in Fig. 7 , for a $0.27 X_{0}$ effective radiator. There is no requirement of a RESH tag in this spectrum. The spectrum shape, with the BGM energy scale set by the $350 \mathrm{GeV}$ electrons, is fitted with the form:

$$
\frac{d n}{d q}=\frac{1}{q^{\alpha}} \times \frac{1}{1+\exp \left[\frac{\left.q-e_{a n d}\right]}{\lambda}\right]}
$$

where $q$ is the total energy of all the photons radiated. In this empirical fit, the $1 / q^{\alpha}$ term reproduces the shape of the thick radiator energy loss spectrum in a Monte Carlo simulation, while the remaining terms produce an easy parametrization of a typical cut off due to the finite energy distribution of the electron beam. Fitted values are given in Table 4.

As can be seen from the fit parameter $e_{e n d}$, there is a slight difference in the 
Table 4: Fit parameters for the energy loss spectrum, with BGM gain set using $350 \mathrm{GeV}$ electrons.

$\begin{aligned} \alpha= & 0.73 \pm 0.02 \\ e_{\text {end }}= & 398 \mathrm{GeV} \pm 3 \mathrm{GeV}, \text { the endpoint of the photon spectrum, related } \\ \lambda & =28 \mathrm{GeV} \pm 1 \mathrm{GeV}, \text { the incident electron energy spread. }\end{aligned}$
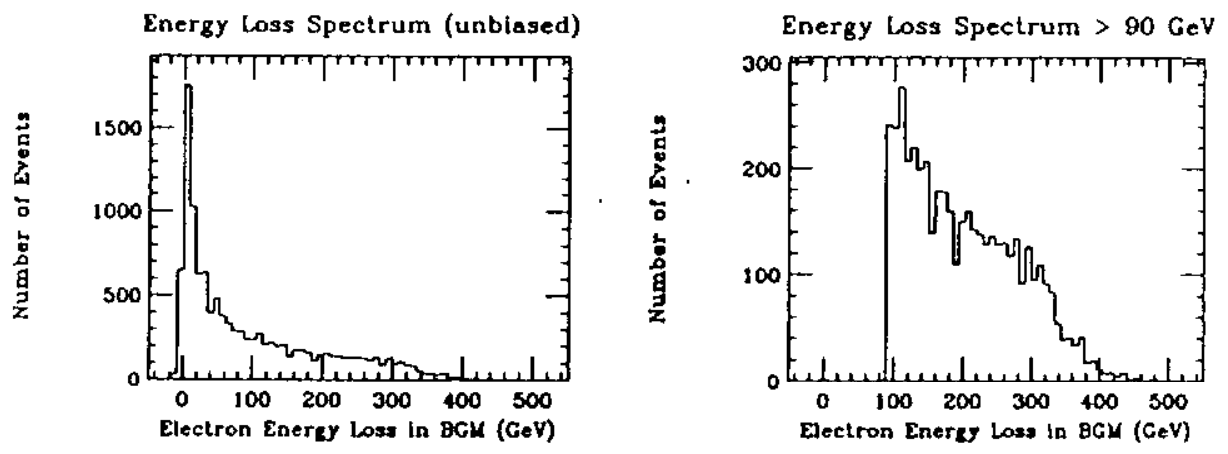

Figure 7: Photon spectrum obtained from $350 \mathrm{GeV}$ electrons for a) all energies; b) energies greater than $100 \mathrm{GeV}$ as measured calorimetrically in the $\mathrm{BGM}$ counter.

BGM response to electrons and photons. It is expected that $e_{e n d}$ also correspond to $350 \mathrm{GeV}$, the average electron energy. In Figures 7 - 10, the BGM energy scale is set using the photon endpoint $e_{\text {erd }}=350 \mathrm{GeV}$.

A 'thick target' bremsstrahlung Monte Carlo calculation predicts $\alpha=0.73$ for the distribution of $q$, the sum of the photon energies or the electron's energy loss for a $0.27 X_{0}$ radiator, in good agreement with the experimental distribution.

Figure 8 shows the tagged (electron energy loss) photon spectrum, integrated over all accepted RESH tagging hit pattern configurations. Figure 9 shows the photon tagging efficiency obtained by dividing the tagged energy loss spectrum without RESH trigger requirement into the same distribution requiring the RESH-LOW trigger to be set. Typical tagged energy loss spectra (measured in BGM) for selected tagging RESH hit patterns are shown in Fig. 10. 


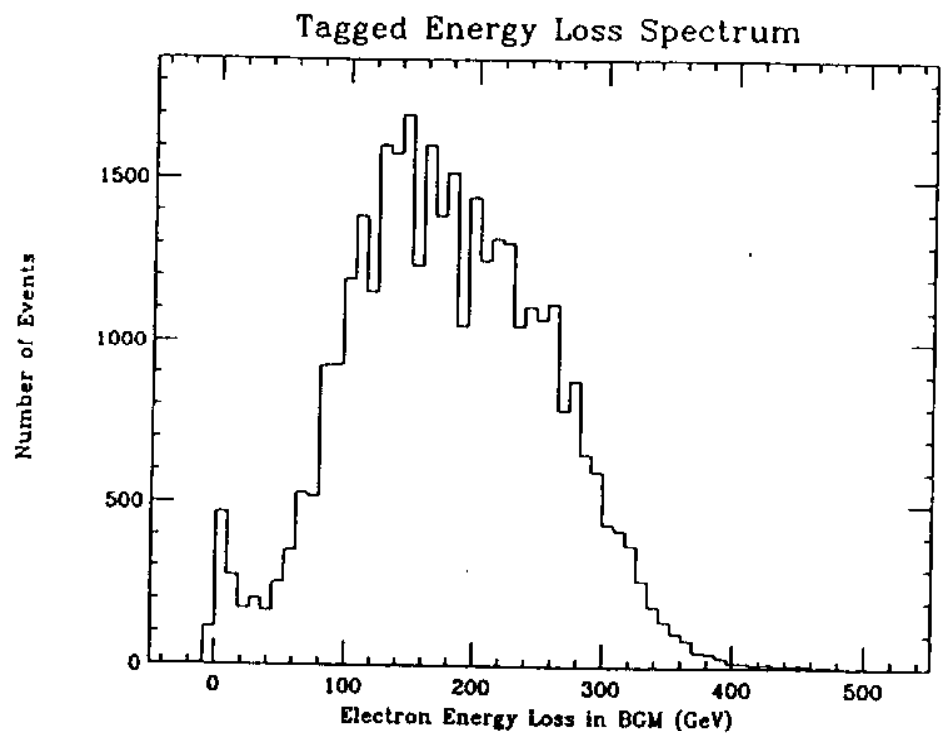

Figure 8: Tagged energy loss spectrum measured calorimetrically in BGM.

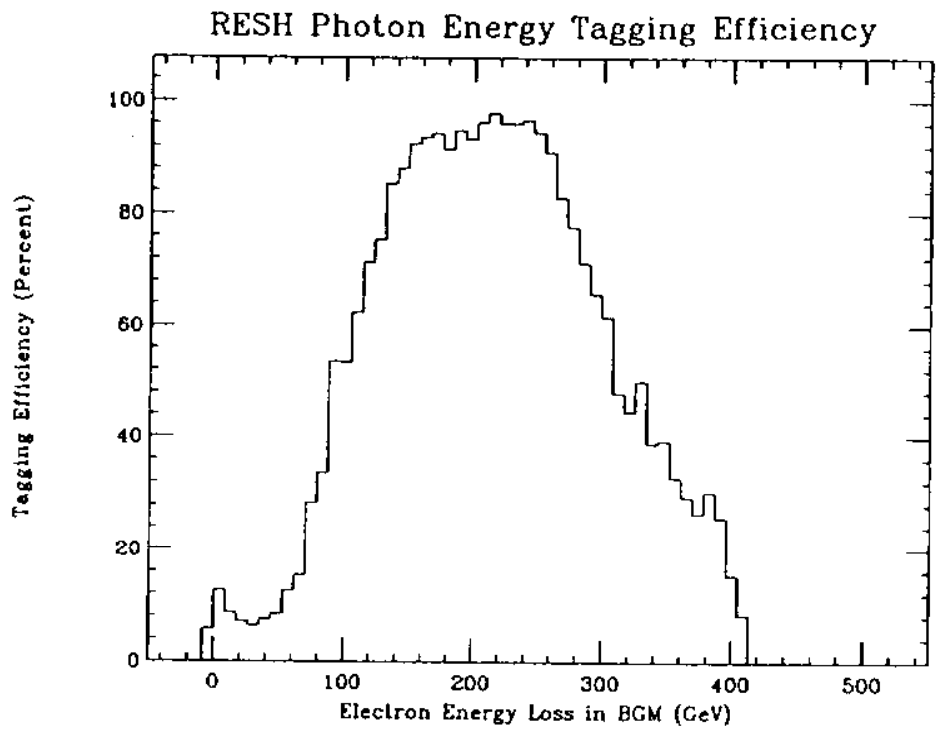

Figure 9: RESH Photon tagging efficiency vs. electron energy loss in BGM. 

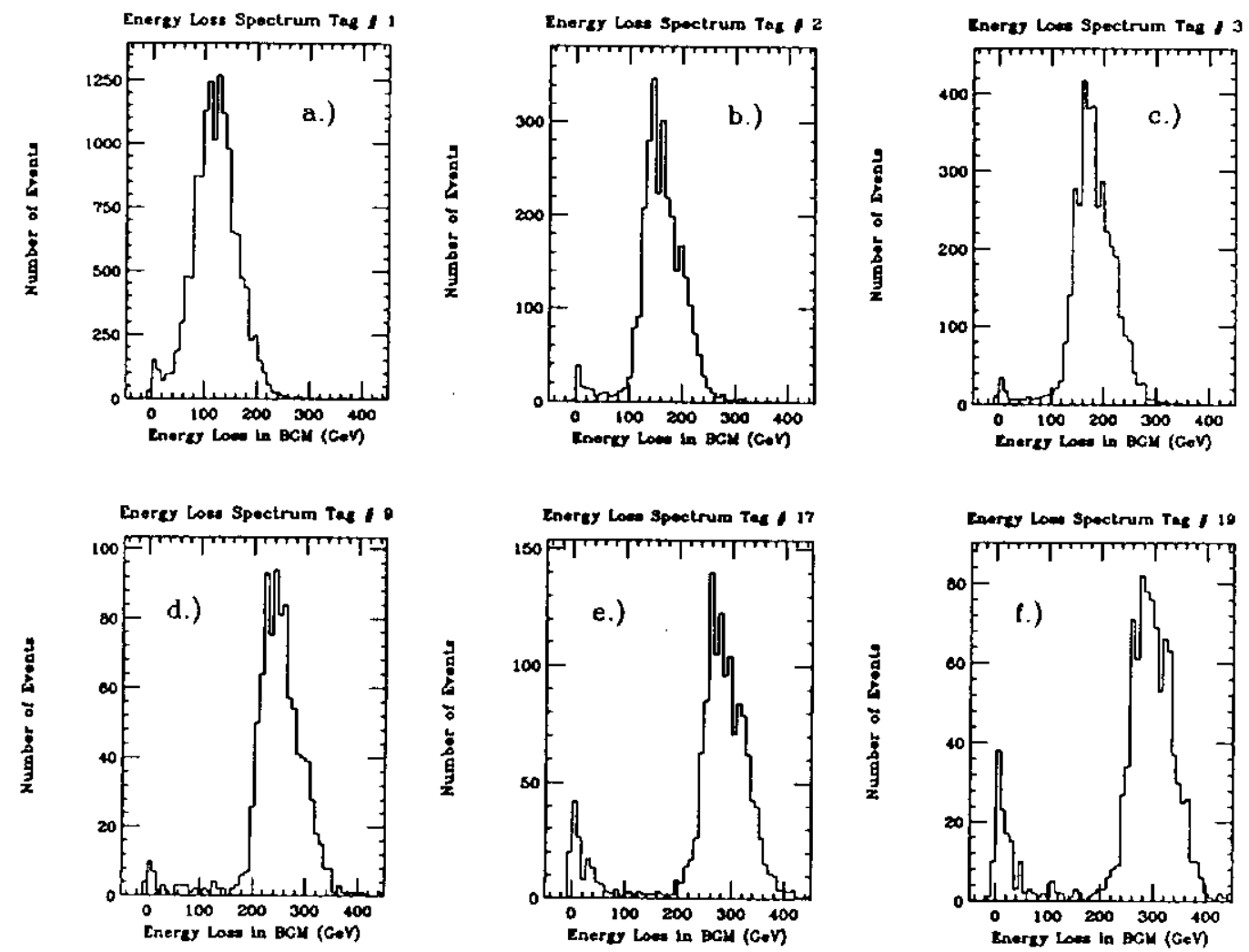

Figure 10: Tagged energy loss spectrum in BGM for various RESH patterns ranging from the lowest energy a) to the highest $f$ ). 

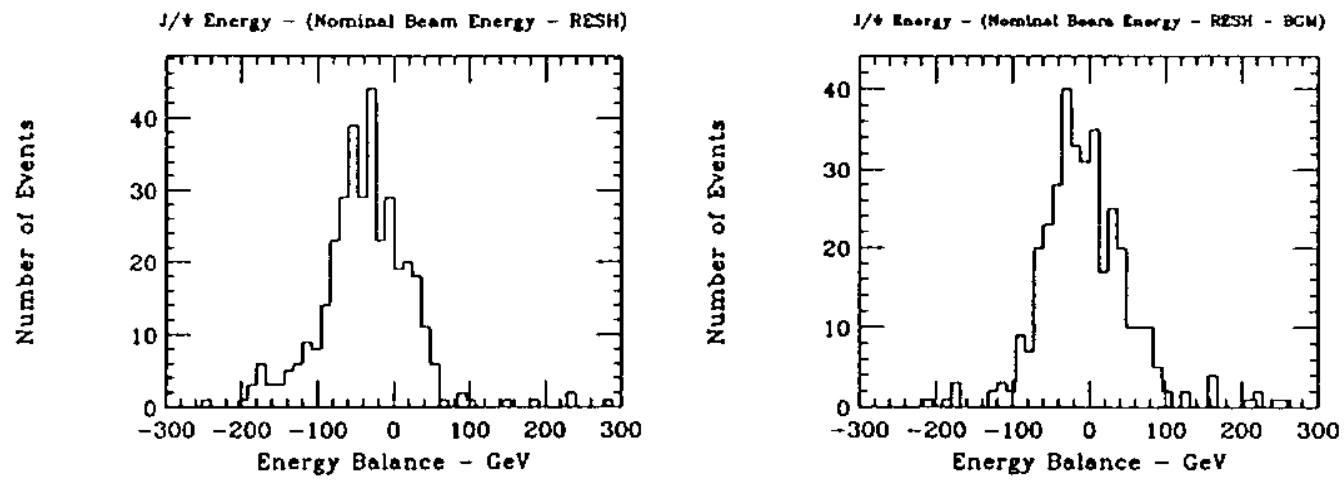

Figure 11: Energy balance between elastic $\mathrm{J} / \psi$ and calorimetric photon energy a) without Beam Gamma correction; b) with Beam Gamma correction.

Figure 11 shows how the RESH and BGM work together in determining the event energy on an event by event basis. Plotted first is the electron energy loss from RESH minus the $J / \psi$ energy for quasi-elastic events determined using the experiment's magnetic spectrometer. The assumed incident electron energy is $350 \mathrm{GeV}$. The energy does not balance due to extra multiple photons that are coincident with the photon producing the $\mathrm{J} / \psi$ but are not accounted for. By subtracting also the summed energies of these non-interacting photons hitting the BGM counter, the energy balance is improved, as shown in the second plot where the energy spread $(\sigma)$ is consistent with the uncorrected beam momentum spread and the tail on the low side has disappeared.

An important problem occurs due to second order processes in the thick radiator. Some of the photons produced can be re-converted into $e^{+} e^{-}$pairs within the radiator. This will either result in the loss of photons that would otherwise strike the experimental target, leading to a mis-measured flux, or produce "false tags" ${ }^{n}$. These false tags can be generated when an incident electron suffers only a small energy loss within the radiator. It is not deflected sufficiently to register in the RESH hodoscope, but strikes the electron dump. A photon radiated by this electron can pair-convert in the radiator, possibly having the electron from 
the pair fall within the RESH acceptance. This is a more serious background as the apparent tagging energy increases. The bremsstrahlung tail near zero energy in the BGM can be seen to increase as the tagging energy increases in Fig. 10 a through Fig. $10 \mathrm{f}$. These backgrounds are identified in later runs with the addition of showet counters at the electron dump and on the positron side of the sweeping magnet.

\section{Incident Beam Magnetic Spectrometer}

As stated in the previous section, the main uncertainty in the energy measurement for the interacting photon is due to the $\pm 50 \mathrm{GeV}$ energy spread of the incident electron beam. To improve the resolution, starting with the 1990 run, an incident beam magnetic spectrometer has been installed[7]. The spectrometer consists of 5 large area microstrip planes which measure the bend angle of the particles as they pass through the two dipoles that form the last leg of the double dogleg. This arrangement is shown in Fig. 12. The design resolution for the incident electron momentum is $2.2 \%$. This resolution is comparable to, but somewhat better than, the resolution on the energy measurement of the electron after it radiates.

In one particular operating mode of the beamline, the so-called 'double band' configuration[11], there are two beams simultaneously, electrons and positrons, which converge at the final pair of beam dipoles, with a mean angle with respect to the theoretical neutral beam of \pm 3 mrad. The beams emerging from the dipoles are parallel and enter a net focusing triplet of quadrupoles. Monte Carlo simulations show that the best performance for the incident beam spectrometer is obtained when the five microstrip detectors are placed symmetrically around the dipoles. Tag 4 and Tag 5, the most downstream planes, are set as far apart as possible, given the geometry of the beam line, Tag 4 just after the second bending magnet and Tag 5 just before the first quadrupole. The two upstream microstrip planes, Tag 1 and Tag 2, are placed symmetrically with respect to the bend center of the two dipoles. The third detector, Tag 3, whose purpose is to provide redundancy to help the pattern recognition in case of multiple beam tracks, spurious noise, or out-of-time hits, is located between the two bending magnets. Increasing the lever arm for the upstream part of the system, which is not constrained by other elements in the beam, would increase the resolution somewhat, at the cost of acceptance. The electron and positron beams are somewhat separated in this part of the system and do not quite fit on the 


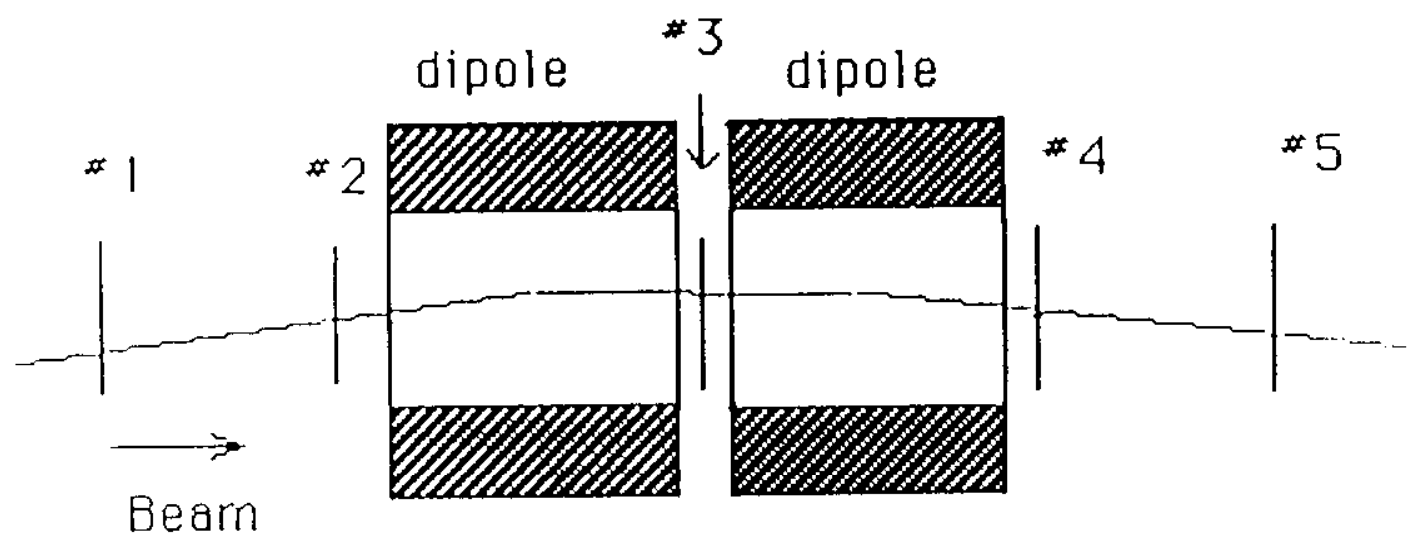

Figure 12: Layout of the beam tagging spectrometer.

upstream detector, even in the chosen compromise configuration. The distance between tag 1 and tag 5 is 13.7 meters and the acceptance is about $81 \%$ for the electrons and positrons whose bremsstrahlung photons reach the experimental target. Each microstrip detector is 300 microns thick with a pitch of 300 microns. Each $7.7 \mathrm{~cm} \times 5.7 \mathrm{~cm}$ detector has 256 channels for a total of 1280 channels. The total field integral is 30.8 kilogauss-meters at a nominal electron beam setting of $350 \mathrm{GeV} / \mathrm{c}$ and is scaled with the beam's central momentum setting.

\subsection{Principle of Operation}

Silicon microstrip detectors are chosen because they can operate in a high intensity beam (in excess of $10^{7}$ particles per second), have an intrinsically fast time response, can easily satisfy the granularity requirement, and are quite robust compared to PWC's or drift chambers.

The Fermilab beam is bunched in narrow RF buckets of about 1 nsec width 


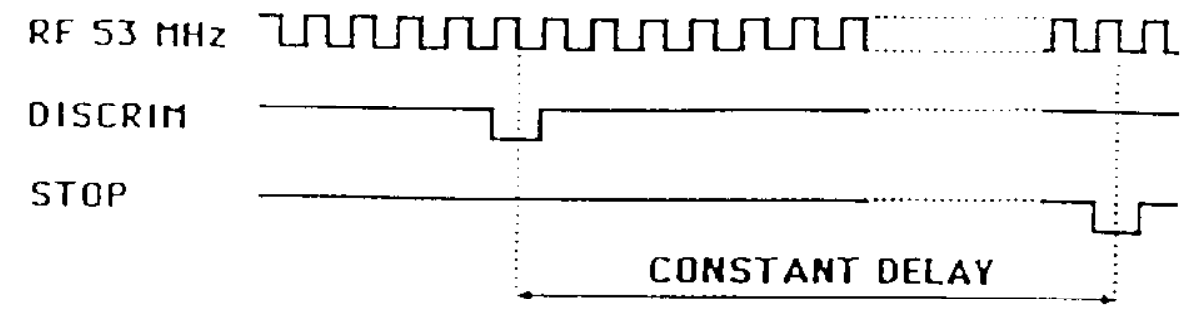

Figure 13: Timing relationships in the beam tagging spectrometer.

with a spacing between successive buckets of $18.8 \mathrm{nsec}$. Because of the high rate, the jitter of the detector pulses with respect to any latching strobe must be kept as small as possible. For this reason, the read out electronics are placed close to the detector to avoid long transmission lines for the 1280 channels.

The trigger from the E687 spectrometer is formed in the counting room and is available at the detector site about $2.5 \mu \mathrm{sec}$ after the beam particle crossed the detector. This means that the readout must store data in a pipeline at least $2.5 \mu \mathrm{sec}$ long so that it will have the right data when a trigger comes.

The principle of the readout and its timing is illustrated in Figure 13. The beam particles have a constant phase with respect to the accelerator RF. This phase is smeared by a few nsec by the jitter introduced by the detectorpreamplifier-discriminator chain. The particle signals, RF/data from the discriminator, are sent to a shift register continuously clocked by the accelerator RF. When a trigger is accepted, a stop signal, RF/stop, is sent from the counting room to the system in the beam line to stop the shift register. Since the trigger decision time is constant, the delay between the discriminator pulse at the input of the shift register and the stop signal is also constant. The beam particle signals corresponding to the trigger are extracted from the memories by reading the proper cell in the shift register at a fixed offset from the stop.

The critical timings of the system are:

1) The RF/data timing: due to the jitter and to incorrect timing, the real hits could be randomly latched in two consecutive time cells of the shift register. 
2) The RF/stop timing: due to incorrect timing, the shift register could be randomly stopped with a \pm 1 cell ambiguity.

\subsection{Electronics}

To minimize the jitter of the data being written into the memories with respect to the RF, fast current preamplifiers with a rise time of less than 10 nsec are used. These are realized on thick film circuits and have a bipolar input. transistor. The noise FWHM is equivalent to $18 \mathrm{KeV}$ in silicon with the detector connected, for a signal-to-noise ratio of 11 .

Threshold discriminators with an independent CAMAC programmable threshold for each channel are employed. The output signal from these discriminators is as wide as the RF period. This is to eliminate the possibility of latching the same signal in more than one memory location once the RF/data timing has been properly set. There is also a $60 \mathrm{nsec}$ protection dead time to prevent the discriminator from being triggered twice by the same particle signal.

Fast ECL circular memories are clocked by the $53 \mathrm{Mhz}$ accelerator RF signal. Since the timing, and location within the memories, of the RF/data for the triggered beam particle is determined by subtracting a constant delay relative to the $R F$ /stop signal, it is very important to maintain timing of both the RF/data and RF/stop to within a fraction of the $18.8 \mathrm{nsec}$ RF period. The total delay between the RF/data input to the memories and the RF/stop consists of three quantities: $a$. the time interval from beam particle traversing the beam momentum spectrometer to the formation of the Level I event trigger (clocked to within \pm 1 nsec by the relativistic particles themselves), b. the time interval necessary to form the Level II trigger after the Level I trigger is formed, and c. the the time interval from the formation of the Level II trigger until the RF/stop signal is received at the local memories (consisting of fixed electronic and cable delays). Although the time interval between Level I and Level II triggers is nominally a constant, a special counter/timer is installed both to measure this trigger decision time in units of the RF clock rate, and to restandardize the timing to within a fraction of the RF period. This counter, clocked by the accelerator RF signal, is started by the Level I trigger and stopped by the coincidence of the clock RF signal and the signal representing the formation of the Level II trigger. This produces an RF/stop signal at the memories without jitter with respect to the RF phase. The time interval to produce the Level II trigger is thus measured and can be used to calculate the overall delay. Operationally, the memories are stopped only upon production of a Level II trigger, thereby 
reducing deadtime.

The data readout is started approximately $50 \mathrm{nsec}$ after the stop arrival. There is a daisy chain connecting the 8 memories in the same plane with a 32 bit data bus to the relative termination unit and the 5 termination units in the system, one per plane. The first termination unit is the master and it is connected to the E687 Fastbus DAQ. While data are being read out, they are also stored inside the relative termination unit and can be accessed via CAMAC. The read-out rate is $5 \mathrm{MHz}$ for 32 bit words and the total read-out time for 1280 channels is $8 \mu \mathrm{sec}$. As soon as the readout is been completed, the read return signal from the last memory restarts the system.

\subsection{Performance}

In Figure 14, electron beam profiles demonstrate that the system is properly set.

The system is aligned geometrically by turning of the bending magnets and taking undeflected electrons and positrons.

The absolute momentum scale is calibrated by using the main E687 spectrometer where the momentum analysis is done with two 1.4 Tesla (maximum) bending magnets, a system of 20 multiwire proportional chambers and a high resolution silicon vertex detector[2]. The spectrometer magnetic field has been accurately mapped and its absolute momentum scale is checked by reconstructing the invariant mass of a large number of narrow resonances, including the $K_{,}^{0}$, the $\Lambda^{0}$, and the charmed $D^{0}$ and $D^{+}$mesons, states whose masses are accurately known.

Low intensity pion runs at $30,60,100,200$ and $350 \mathrm{GeV}$ are taken with the radiator and the experiment target removed. Only one track is required in both the incident beam spectrometer and the $\mathrm{E} 687$ spectrometer and the difference between the momenta reconstructed by the two systems is compared. The field in the beam spectrometer magnet is scaled with the pion energy, so that the beam spectrometer resolution (apart from MCS effects that have been taken into account) is energy independent. At $60 \mathrm{GeV}$ the overall $2.5 \%$ resolution on the momentum difference is dominated by the beam spectrometer resolution, as shown in Figure 15, while at $350 \mathrm{GeV}$ it is $6.5 \%$, dominated by the E687 spectrometer resolution. (The spectrometer magnet current is NOT scaled with energy but remains fixed at its normal data-taking value where the magnetic field was mapped and calibrated.) The incident electron momentum resolution, after unfolding the spectrometer resolution, is found to be $2.2 \%$, independent of 

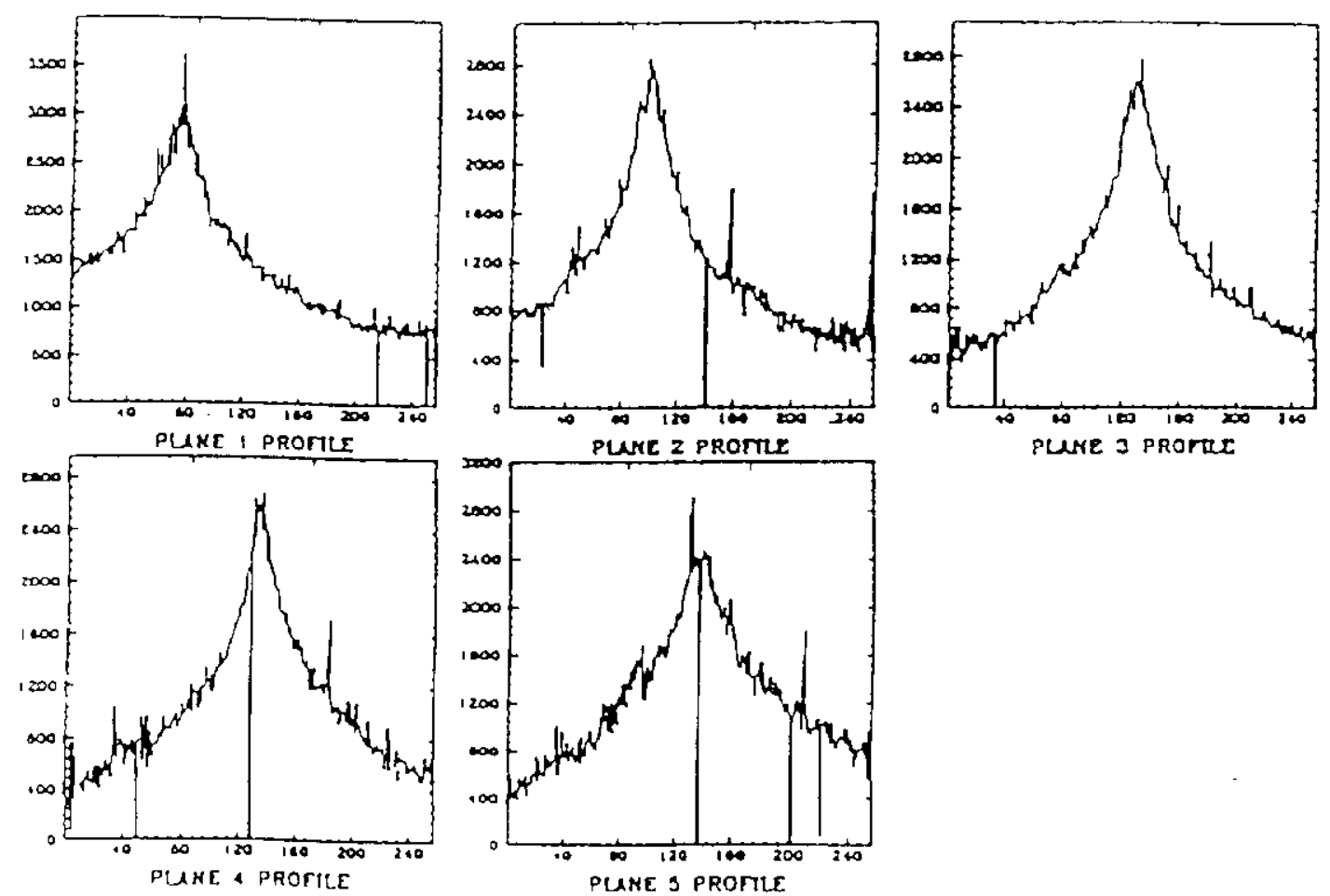

Figure 14: Electron beam profiles in the beam momentum tagging spectrometer planes. 
energy.

Efficiency studies are done for each strip using tracks within the acceptance. The average efficiency per plane is about $98 \%$ taking into account dead electronics channels and disconnected strips.

\subsection{Electron Beam Energy Spectrum}

A typical electron beam energy spectrum measured with the beam momentum tagging spectrometer is shown in Figure 16. This spectrum was taken using electrons under the slightly different 'double-band' beam optics configuration[11] with an average electron momentum setting of $320 \mathrm{GeV} / \mathrm{c}$ during the 1990-91 running periods.

\section{Improvement to Primary Target}

After the first run of E687, it became clear that the experiment could handle more photons than the bearn could provide. In the initial beam design, beryllium was chosen as the production target because it was simple to implement. After the first run, there was a comparative study with targets composed of beryllium, deuterium, and lithium.

The highest photon yield is obtained when the greatest number of primary protons interact in the target, producing neutral pions which in turn decay to two photons. The photons can convert to $e^{+} e^{-}$pairs as they pass thru the target. Therefore, the target material should have the smallest possible ratio of radiation length to interaction length.

There are three important factors which affect the photon yield. First and foremost is the ratio of interaction length, $\lambda$, to the radiation length, $\mathrm{X}_{0}$ of the target material. Second is the " $A$ " dependence as a function of $x_{f}[12]$. Per nucleon, there are more high $x_{f}$ pions produced in light nuclei than in heavier nuclei. Third is the beamline acceptance. When a longer target, such as a liquid with cryostat, is used, its centerline must be placed slightly farther from the first quadrupoles, which reduces the acceptance of the beam.

The GEANT[13] Monte Carlo program is used to simulate the production and absorption of photons in various target materials. Production of photons by the intereactions of secondary hadrons is included in this calculation. Figure 17 shows the predicted number of photons with energy greater than 250 $\mathrm{GeV}$ per incident $800 \mathrm{GeV}$ proton as a function of target length for deuterium, lithium, and beryllium. Deuterium has a clear advantage. Due to the effects 

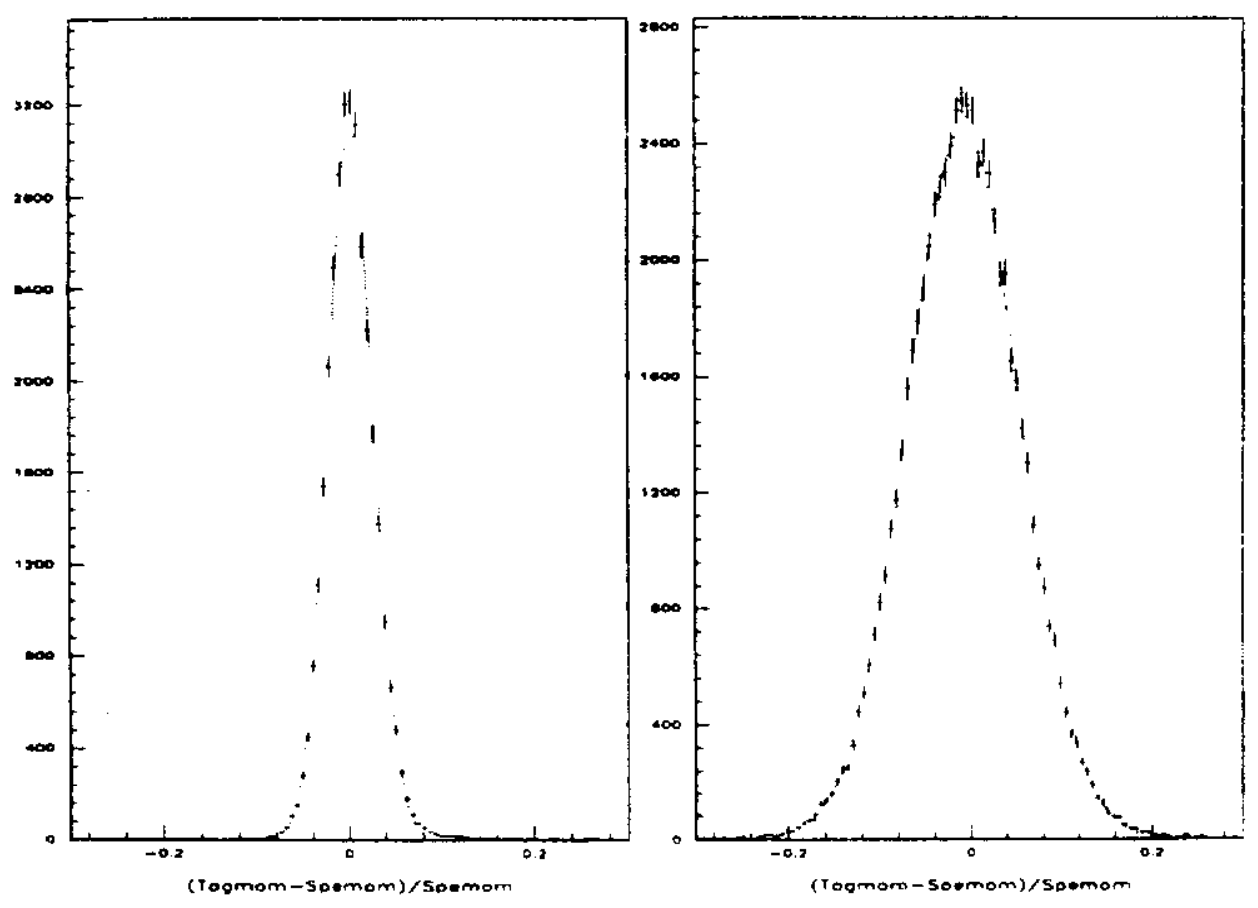

Figure 15: The quantity $\left(p_{t a g}-p_{\text {opec }}\right) / p_{\text {opec }}$ for a) $60 \mathrm{GeV} / \mathrm{c}$ pions and b) 350 $\mathrm{GeV} / \mathrm{c}$ pions. 


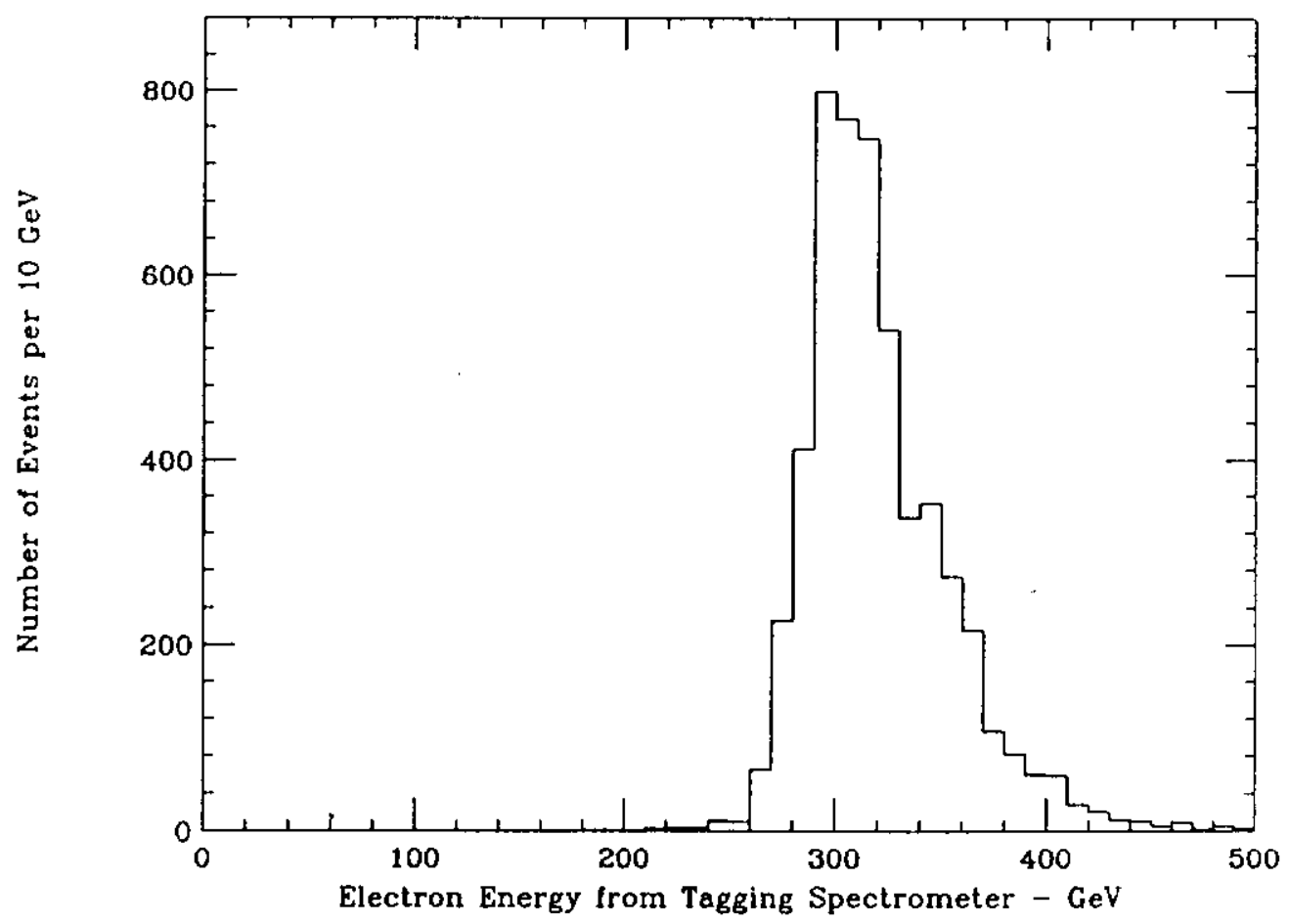

Figure 16: Typical electron beam energy spectrum measured with beam momentum tagging spetrometer. 
of acceptance for a long target, the optimum yield is reduced by $14 \%$ and the optimum target length changed from 1.6 to 1.0 interaction length.

Based on this study, in 1990 the primary production target was changed to a 3.5 meter long liquid deuterium target. The drawbacks of a liquid deuterium target are the physical length and the required cryogenics. The deuterium target does, in fact, increase the photon yield by a factor of 1.5. The target reliability has proven to be excellent. 


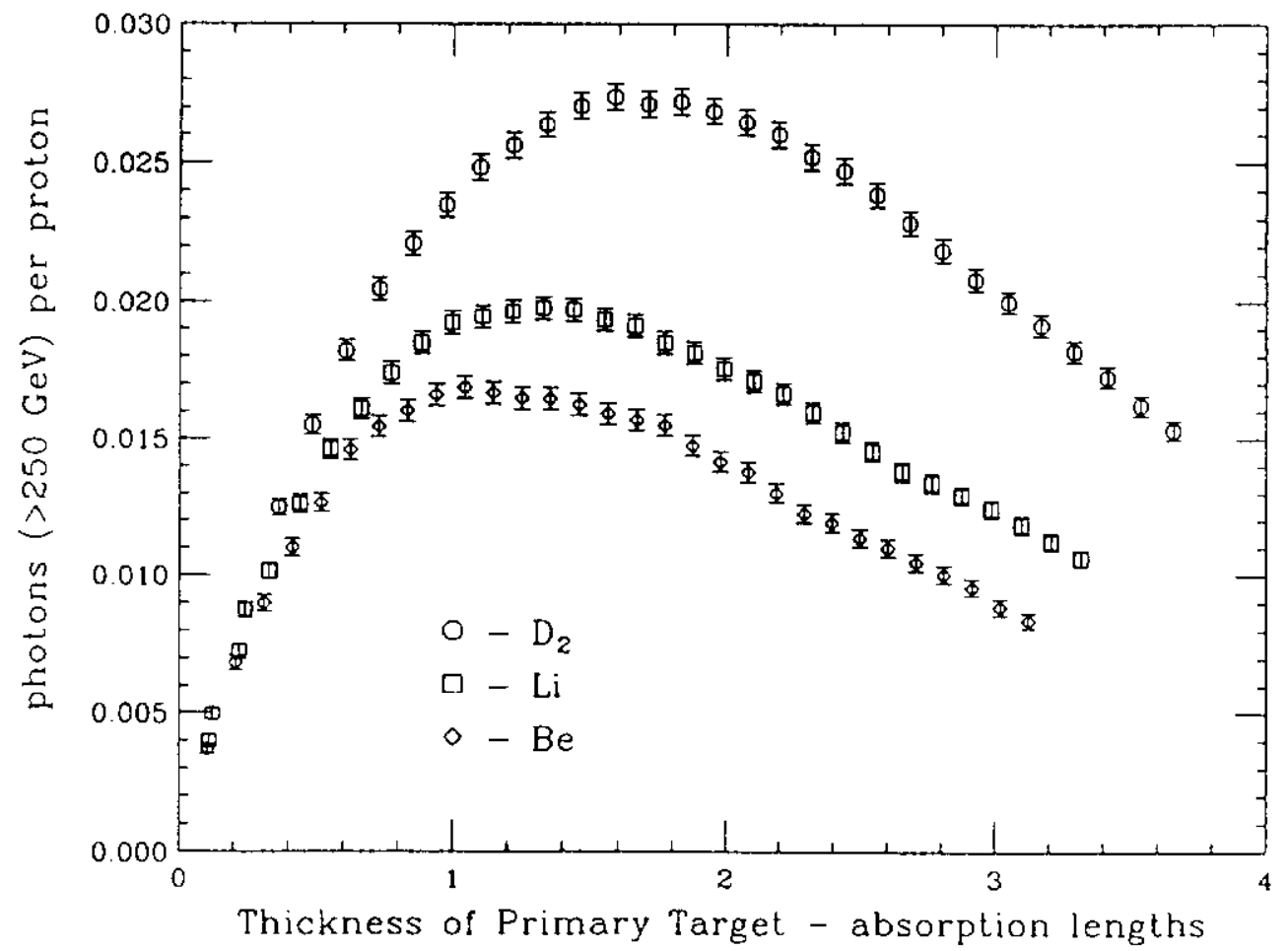

Figure 17: Calculated relative photon yields for beryllium, lithium, and deuterium targets. 


\section{Acknowledgements}

It takes many people to construct a facility such as the Fermilab Wide. band Beam/E687 spectrometer. We wish to acknowledge the assistance of the staffs of Fermilab, Laboratori Nazionali di Frascati, the physics departments of the University of Colorado, University of Illinois, Northwestern University, University of Notre Dame, the I.N.F.N. Sections, the Physics Departments of the Bologna University, Milano University, Pavia University and the Italian Ministero dell'Università e della Ricerca Scientifica e Tecnologica.

We would like to thank Ken Stanfield and John Peoples of Fermilab for encouraging and supporting the eftort from its very beginning. Tom Kirk, the Tevation II project manager, likewise provided essential support and assistance to this effort. We want to thank the Fermilab Research Division for the design, construction, and installation activities associated with this large project. We owe much to Jon Hawkins who served as beam line physicist during the design stage and did much of the work on the beam optics. Lou Kula and Fred Browning did much of the initial design work and cost estimates for the proposal. Al Guthke supervised the installation, vacuum work, and provided invaluable advice and assistance on many aspects of the project. Rich Stanek supervised all the cryogenic work. Mike Gerardi, the radiation safety officer, developed a design for the shielding and interlocks which has satisfied, virtually without modifications, the extremely rigorous review recently completed by Fermilab using standards that were more strict than those in place at the time of the design. Age Visser designed the electrical power distribution systems which have served so well for so long. Leon Beverly and Dwight Featherston supervised the electrical installations. These people and their dedicated and talented staffs deserve the credit for the successful operation of the Wideband Beam. We want to especially thank the Research Division Operations Group for the skill with which they tuned and operated this very complex beam. We would also like to thank the Accelerator Division for the excellent performance of the machine during the run.

This research was supported in part by the Istituto Nazionale di Fisica $\mathrm{Nu}$ cleare (I.N.F.N.) of Italy, Ministero della Università e della Ricerca Scientifica e Tecnologica, the National Science Foundation, and the U.S.Department of Energy. 


\section{References}

[1] L.M. Jones and H.W. Wyld, Phys.Rev. D17, 759(1978).

[2] P.L. Frabetti, et al., "Description and performance of the Fermilab E687 spectrometer", Nucl. Instr. and Meth. A320(1992) 519.

[3] C. Halliwell et al., Nucl. Instr. and Meth. 102(1972)51,

D. Aston et al., Nucl. Instr. and Meth. 197(1982)287,

M. Winter, "Production du Faisceau de Photons et Determination en Linge de leur Energie dans l'Experience de Photoproduction NA14", Universite Louis Pastuer de Strasbourg, thesis, 1981, CRN/HE 81-03,

H.W. Atherton, et al., "Electron and Photon Beams in the SPS Experimental Areas", CERN/SPS 85-43 (EBS).

[4] J. Butler, et al., "Design for a New Wide-Band Neutral Beam for the Tevatron", Fermilab TM-963 (1980), (updated 1989).

[5] D. Kruse and G. Koizumi, Fermilab TM-607 (1975) unpublished, and S. Snowdon and H. Stredde, Data Sheet 2220.10, Fermilab, unpublished.

[6] E. Kneip, Fermilab Technical Specification 2221-ES-27487 (1974) and S. Snowdon, Data Sheet 10-28-74, Fermilab, unpublished.

[7] G. Alimonti et al.," A momentum tagging system for the wide band $e^{+} / e^{-}$ beam at FNAL", Nucl. Instr. and Meth. A314(1992)411.

[8] Accumulator Dipoles: "Design Report, Tevatron I Project, Fermilab, Batavia, IL, September 1984".

[9] C.R. Kerns, "A high-rate phototube base", IEEE Trans. Nucl. Sci., Vol. NS-24, No. 1, pp 353-355, Feb. 1977.

[10] J. Knauer, et al., Proceedings of the Calorimeter Workshop, Fermilab, 1975, page 215, unpublished.

[11] P. Kasper, et al., "Wide Band to 'Double Band' Upgrade", Fermilab TM$1552(1988)$.

[12] D.S. Barton et al., Phys. Rev. D27, 2580(1983).

[13] R. Brun, et al., "GEANT3", CERN Data Handling Division DD/EE/841,1987 . 